\title{
Networked Control of Distributed Energy Resources: Application to Solid Oxide Fuel Cells
}

\author{
Yulei Sun, Sathyendra Ghantasala and Nael H. El-Farra ${ }^{\dagger}$ \\ Department of Chemical Engineering \& Materials Science \\ University of California, Davis, CA 95616 USA
}

\begin{abstract}
This paper presents a model-based networked control approach for managing Distributed Energy Resources (DERs) over communication networks. As a model system, we consider a solid oxide fuel cell (SOFC) plant that communicates with the central controller over a bandwidthconstrained communication network that is shared by several other DERs. The objective is to regulate the power output of the fuel cell while keeping the communication requirements with the controller to a minimum in order to reduce network utilization and minimize the susceptibility of the SOFC plant to possible communication disruptions in the network. Initially, an observer-based output feedback controller is designed to regulate the power output of the SOFC plant at a desired set-point by manipulating the inlet fuel flow rate. Network utilization is then reduced by minimizing the rate of transfer of information between the fuel cell and the supervisor without sacrificing stability or performance. To this end, a dynamic model of the fuel cell is embedded in the supervisor to approximate the dynamics of the fuel cell when measurements are not transmitted by the sensors, and the state of the model is updated using the observer-generated state estimate that is provided by the SOFC plant sensors at discrete time instances. An explicit characterization of the maximum allowable transfer time between the sensor suite of the fuel cell and the controller (i.e., the minimum allowable communication rate) is obtained in terms of model uncertainty and the choice of the control law. The characterization accounts for both stability and performance considerations. Finally, numerical simulations that demonstrate the implementation of the control architecture and its disturbance handling capabilities are presented.
\end{abstract}

\section{INTRODUCTION}

Distributed Energy Resources (DERs) are a suite of onsite, grid-connected or stand-alone technology systems that can be integrated into residential, commercial, or institutional buildings and/or industrial facilities. These energy systems include distributed generation, renewable energy sources, and hybrid generation technologies; energy storage; thermally activated technologies that use recoverable heat for cooling, heating, or power; transmission and delivery mechanisms; control and communication technologies; and demand-side energy management tools. Such distributed resources offer advantages over conventional grid electricity by offering end users a diversified fuel supply; higher power reliability, quality, and efficiency; lower emissions and greater flexibility to respond to changing energy needs.

As the number and diversity of DERs on the grid increases, dispatching these resources at the right time and

To whom correspondence should be addressed: E-mail: nhelfarra@ucdavis.edu. Financial support by the UC Energy Institute is gratefully acknowledged. accounting for the flow of energy correctly become complex problems that require reliable monitoring and telemetering equipment, as well as reliable communication and control between DERs and loads. A distributed power network with thousands of small generators requires far more sophisticated communications and control systems than a radial grid focused on a few big plants. Traditional supervisory control and data acquisition systems with centralized control rooms, dedicated communication lines, and specialized operators, are not cost effective to handle a large number of DERs spread over the grid. Advanced communication and control technologies are needed to enable integration and interoperability functions of a broad range of DERs. These technologies offer a digitally controlled, "smart" electricity network with broad-band communication capabilities. According to some estimates [1], the market potential for advanced control and communications technologies in managing DERs (based on 5-10\% energy savings achieved) is between $\$ 3.75$ billion and $\$ 7.5$ billion domestically, and between $\$ 15$ billion and $\$ 30$ billion worldwide.

While managing DERs over a communication network offers an appealing modern solution to the control of distribute energy generation, it poses a number of challenges that must be addressed before the full economic and environmental potential of DERs can be realized. These challenges stem in part from the inherent limitations on the information transmission and processing capabilities of communication networks, such as bandwidth limitations, network-induced delays, data losses, signal quantization and real-time scheduling constraints, which can interrupt the connection between the central control authority (the supervisor), the generation units and the loads, and consequently degrade the overall control quality if not properly accounted for in the control system design. Despite the availability of fast and reliable communication networks, the fact that the distributed power market is primarily driven by the need for super-reliable, high-quality power implies that the impact of even a brief communication disruption (e.g., due to local network congestion or server outage) can be substantial. In sites such as hospitals, police stations, data centers and high-tech plants which cannot afford blackouts, millisecond outages that merely cause lights to flicker will cause costly computer crashes. For example, First National Bank of Omaha puts the cost of outages at its credit card processing center, seventh largest in the nation, at $\$ 100,000$ per minute [2]. Such high-stakes risks provide 
a strong incentive for the development of robust control and communication strategies that achieve the desired levels of power supply and quality from DERs while minimizing the reliance on the communication medium, which in turn minimizes the impact of data losses and disruptions on the power supply.

Over the past decade, several efforts have been made towards the development and implementation of control strategies for DERs (e.g., [3], [4], [5], [6], [7], [8]). Important contributions in this direction include the use of conventional and model-based feedback control algorithms to regulate various types of grid-connected DERs in order to enhance power system stability (e.g., [9], [10], [11], [12]), mitigate power quality problems (e.g., [13]) and improve the continuity of electricity supply (e.g., [14]), as well as the development of various distributed control and coordination architectures using multi-agent based control approaches (e.g., [15], [16], [17], [18]). While the focus of these studies has been mainly on demonstrating the feasibility of the developed control algorithms, the explicit characterization and management of communication constraints in the formulation and solution of the DER control problem have not yet been addressed.

Motivated by these considerations, we focus in this work on the problem of controlling DERs over communication networks. As a model system, we consider a solid oxide fuel cell (SOFC) plant that communicates with the central controller over a bandwidth-constrained communication network that is shared by many other DERs. A model-based network control approach is presented to regulate the power output of the SOFC plant while keeping the communication requirements with the supervisor to a minimum. The rest of the paper is organized as follows. Following an overview of the problem formulation and solution methodology in Section II, a SOFC model is presented in Section III and used in Sections IV to design the networked control structure. An explicit characterization of the minimum allowable rate at which the sensors of the fuel cell must communicate with controller to maintain the desired closed-loop stability and performance properties is obtained, and numerical simulations that demonstrate the efficacy of the networked control architecture are presented. Finally, concluding remarks are given in Section $\mathrm{V}$.

\section{PRoblem FORMUlation AND SOLUtion METHODOLOGY}

We consider an array of DERs managed by a higherlevel supervisor over a bandwidth-limited communication network as depicted in Figure 1. Each DER is modeled by a continuous-time system with the following state-space description:

$$
\dot{x}_{i}=f_{i}\left(x_{i}\right)+G_{i}\left(x_{i}\right) u_{i}, \quad y_{i}=h_{i}\left(x_{i}, u_{i}\right)
$$

where $x_{i} \in \mathbb{R}^{n_{i}}$ denotes the vector of state variables associated with the $i$-th DER (e.g., exhaust temperatures and rotation speed in turbines and internal combustion engines, operating temperature and pressure in fuel cells), $y_{i} \in \mathbb{R}^{q_{i}}$ is the vector of measured and/or controlled outputs (e.g., output power, voltage and frequency), $u_{i} \in$ $\mathbb{R}^{m_{i}}$ denotes the vector of manipulated inputs associated with the $i$-th DER (e.g., inlet fuel flow rate in fuel cells, shaft speed in turbines), and $f_{i}(\cdot), G_{i}(\cdot)$ and $h_{i}(\cdot)$, are smooth nonlinear functions. In the hierarchical structure of Figure 1, each DER has local, on-board sensors and actuators with some limited built-in intelligence that gives the DER the ability to run autonomously for periods of time when no communication exists with the remote software controller (the supervisor). The local sensors in each DER transmit their data over a shared communication channel to the supervisor where the necessary control calculations are carried out and the control commands are sent back to each DER over the communication network. Based on load changes, changes in utility grid power prices and the state and capacity of each DER, the supervisor coordinates local power generation. The supervisor is also responsible for monitoring the operational health of DERs, issuing alarms and shutting down DERs if alarms go unheeded by operators, automatically scheduling and dispatching DERs in an economically optimal manner. One

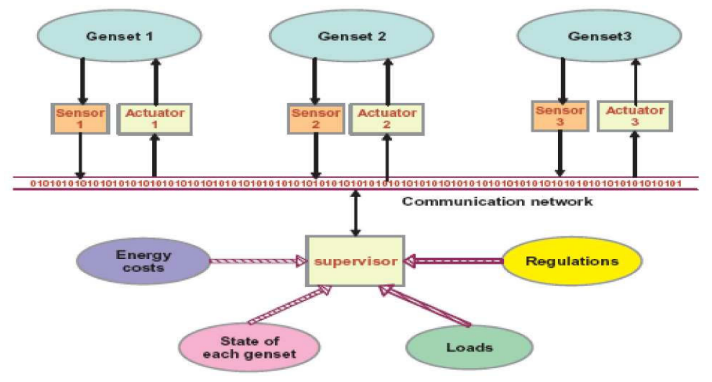

Fig. 1. Management of DERs over a shared communication network.

of the main problems to be addressed when managing a large number of DERs over a communication network is the large amount of bandwidth required by the different subsystems sharing the communication channel. Optimal control and coordination between the different DERs to meet changes in power demand is best achieved when information (e.g., measurements, control commands) flow continuously between each DER and the supervisor. In traditional control architectures, the dedicated point-to-point connections make the information available continuously. In a networked control system, on the other hand, the feedback path is a network which typically has limited bandwidth and transfers information in a discrete fashion. A tradeoff typically exists where maximal control performance requires frequent communication, while minimal network resource utilization necessary to save on communication costs favors limited communication. Proper characterization and management of this tradeoff is an essential first step to the design of resource-aware networked control and communication strategies that ensure the desired performance 
while respecting inherent constraints on the resources of the communication medium. To address this problem, we will focus in this work on minimizing the sensor-controller communication costs under the assumption that the actuators and supervisor are collocated (i.e., the network exists between the sensors and the controller; generalizations to account for actuator-controller communication constraints are possible and the subject of other research work). To this end, we will consider the following approach:

- Initially design for each DER an appropriate feedback control law that regulates its output (in the absence of communication constraints) at the desired set-point decided by the supervisor.

- To overcome bandwidth constraints in the shared communication network, reduce the collection and transfer of information between each DER and the supervisor as much as possible to limit the bandwidth required from the network and free it for other tasks (e.g., other control loops using the network and/or non-control information exchange) without sacrificing the desired stability and performance properties.

- Obtain an explicit characterization of the maximum allowable transfer time between the sensor suite of each DER and the controller, which is the time between information exchanges. In general this time is different for each DER and depends on the degree of mismatch between the dynamics of each unit and the model used to describe it. For example, if the model describing the behavior of a given DER is accurate, the maximum allowable update period for this DER can be arbitrarily large since there will be no need to communicate measurements in this case.

The following sections demonstrate the application of this methodology on a solid oxide fuel cell plant example.

\section{APPLICATION TO A SOLID OXIDE FUEL CELL}

Fuel cells are important distributed resources due to their high efficiency, low levels of noise and environmental pollution, and flexible modular designs that match versatile demands of customers. As an illustrative example, in this work we consider a stack of solid oxide fuel cells (SOFC) as a DER in a power distribution system.

\section{A. SOFC Model}

A solid oxide fuel cell is an electrochemical device that generates electrical energy from chemical reactions. It consists of two porous electrodes, an anode and a cathode in contact with a solid metal oxide electrolyte between them. Hydrogen rich fuel is fed along the surface of the anode where it releases electrons that migrate externally towards the cathode. The electrons combine with oxygen in air that is fed along the surface of the cathode to form oxide ions. These ions diffuse through the electrolyte towards the anode where they combine with the $H^{+}$ions to form water. The Nernst equation describes the potential difference between the electrodes that drives the reaction and the movement of electrons, and is given by:

$$
\triangle E=\left[\triangle E_{0}+\frac{R T}{2 F} \ln \frac{p_{H_{2}} p_{\mathrm{O}_{2}}^{(0.5)}}{p_{\mathrm{H}_{2} \mathrm{O}}}\right]
$$

where $\triangle E_{0}$ is the standard cell potential, $F$ is Faraday's constant and $p_{\mathrm{H}_{2}}, p_{\mathrm{O}_{2}}, p_{\mathrm{H}_{2} \mathrm{O}}$ are the partial pressures of hydrogen, oxygen and steam respectively. Typically, a number of these cells are connected in series to form a stack, which can be used as a stand alone DER. The overall stack voltage is then given by:

$$
V_{s}=N_{0} \triangle E-r_{0} \exp \left[\alpha\left(\frac{1}{T_{s}}-\frac{1}{T_{0}}\right)\right] I
$$

where $N_{0}$ is the number of cells in the stack, $r_{0}$ is the internal resistance at $T_{0}, \alpha$ is the resistance slope, and $I$ is the load current. In Eq.2, only ohmic losses are included, while activation and concentration losses are neglected.

Under standard modeling assumptions, a dynamic model of the following form can be derived [19] for the SOFC stack from material and energy balances:

- Species balances:

$$
\begin{aligned}
\dot{p}_{H_{2}} & =\frac{T_{s}}{\tau_{H_{2}} T K_{H_{2}}}\left(q_{H_{2}}^{i n}-K_{H_{2}} p_{H_{2}}-2 K_{r} I\right) \\
\dot{p}_{O_{2}} & =\frac{T_{s}}{\tau_{\mathrm{O}_{2}} T K_{\mathrm{O}_{2}}}\left(q_{\mathrm{O}_{2}}^{i n}-K_{\mathrm{O}_{2}} p_{\mathrm{O}_{2}}-K_{r} I\right) \\
\dot{p}_{\mathrm{H}_{2} \mathrm{O}} & =\frac{T_{s}}{\tau_{\mathrm{H}_{2} O} T K_{\mathrm{H}_{2} O}}\left(q_{\mathrm{H}_{2} \mathrm{O}}^{i n}-K_{\mathrm{H}_{2} O} p_{\mathrm{H}_{2} \mathrm{O}}+2 K_{r} I\right)
\end{aligned}
$$

- Energy balance:

$$
\begin{aligned}
\dot{T}_{s} & =\frac{1}{m_{s} C_{p s}} \sum q_{i}^{\text {in }} \int_{T_{r e f}}^{T_{i n}} C_{p, i}(T) d T \\
& -\sum q_{i}^{\text {out }} \times \int_{T_{\text {ref }}}^{T_{\text {in }}} C_{p, i}(T) d T-\dot{n}_{H_{2}}^{r} \triangle \hat{H}_{r}^{o}-V_{s} I
\end{aligned}
$$

where, $i: \mathrm{H}_{2}, \mathrm{O}_{2}, \mathrm{H}_{2} \mathrm{O}, p_{i}$ is the partial pressure of component $i, T_{s}$ is the stack temperature, $q_{i}^{i n}$ is the inlet molar flow rate of component $i, m_{s}$ and $C_{p s}$ are the mass and average specific heat of fuel cell materials excluding gases, $C_{p, i}$ is the specific heat of gas component $i, \triangle \hat{H}_{r}^{o}$ is the specific heat of reaction, $I$ is the load current, $\tau_{i}:=$ $V / K_{i} R T$ is a time constant for $i$-th component, $K_{i}$ is the valve molar constant for component $i$, and $K_{r}=N_{0} / 4 F$. The values for the various model parameters can be found in [19]. Throughout the paper, the system of Eqs.3-4 will be referred to as the SOFC plant.

\section{B. Control problem formulation for the SOFC plant}

Referring to the SOFC plant of Eqs.3-4, the control objective is to regulate the power output of the fuel cell stack at a desired set-point by manipulating the inlet fuel flow rate. The set-point is assumed to be determined by the supervisor based on its knowledge of the load changes in the distributed power network that it manages (this typically requires solving an optimization problem in realtime to coordinate power generation between the DERs and 
determine the optimal set-point for each one; and is beyond the scope of the current work). Measurements from the SOFC plant are collected and sent to the central controller where the control action is calculated and sent back to the actuator to effect the desired change in power output. To simplify the controller design and implementation, we consider the problem on the basis of the linearization of the fuel cell plant around the desired set-point. Linearizing the plant model around the desired steady state yields:

$$
\dot{x}=A x+B u
$$

where $x$ and $u$ are the state and manipulated input vectors for the plant, respectively, defined by:

$$
x=\left[\begin{array}{c}
x_{H_{2}}-x_{H_{2}}^{s} \\
x_{\mathrm{O}_{2}}-x_{\mathrm{O}_{2}}^{s} \\
x_{\mathrm{H}_{2} \mathrm{O}}-x_{\mathrm{H}_{2} \mathrm{O}}^{\mathrm{C}_{2}} \\
T_{s}-T_{s}^{s}
\end{array}\right], u=q_{H_{2}}^{i n}-q_{H_{2}}^{i n, s},
$$

where $x_{i}$ is the mole fraction of component $i$, the superscript $s$ denotes the steady state values of the corresponding states and input, $A, B$ are constant matrices given by:

$$
A=\left[\begin{array}{cccc}
-0.035 & 0 & 0 & 0 \\
0 & -0.314 & 0 & 0 \\
0 & 0 & -0.012 & 0 \\
-8.928 & -28.673 & -3.257 & -0.011
\end{array}\right]
$$

and $B=\left[\begin{array}{llll}0.0035 & 0 & 0 & 0.635\end{array}\right]^{T}$.

In this section, we investigate the output feedback control problem where the full-state of the fuel cell plant is unavailable for measurement and only the stack temperature can be transmitted from the fuel cell to the controller. To address the problem, a state observer of the following form is designed and embedded within the sensor:

$$
\dot{\bar{x}}=(\widehat{A}-L C) \bar{x}+\widehat{B} u+L y
$$

where $\bar{x}$ is the observer-generated estimate of $x, \widehat{A}$ and $\widehat{B}$ are estimates of $A$ and $B$, respectively, $L$ is the observer gain (chosen as $L=\left[\begin{array}{lllll}0 & 0.0041 & -0.007 & 0.8008\end{array}\right]^{T}$ in the simulations), $y$ is the measured output of the plant defined as $y=T_{s}-T_{s}^{s}$, and $C=\left[\begin{array}{llll}0 & 0 & 0 & 1\end{array}\right]$. Then to regulate the power output of the fuel cell in the absence of communication constraints, a stabilizing feedback controller of the form $u=K \bar{x}$, where $K=\left[\begin{array}{llll}-18.4 & 0 & 0 & 0.047\end{array}\right]$, is designed to enhance the speed at which the fuel cell meets the desired power demand from the supervisor. Feedback control is also needed to maintain robust operation in the presence of disturbances.

In the next section, we describe how the output feedback control strategy is tailored to take communication constraints in the sensor-controller link explicitly into account.

\section{NETWORKED CONTROLLER DESIGN AND IMPLEMENTATION UNDER OUTPUT FEEDBACK CONTROL}

When considering a networked control system of the type depicted in Figure 1, state information from the SOFC plant can be received by the controller only through the network. In order to reduce network usage, we embed a dynamic model of the fuel cell in the supervisor to provide it with an estimate of the evolution of the states of the fuel cell when measurements are not available. The use of a model at the controller/actuator side to recreate the dynamics of the fuel cell allows the on-board sensors of the fuel cell to transmit their data at discrete time instances and not continuously (since the model can provide an approximation of the fuel cell dynamics) thus allowing conservation of network resources. The computational load associated with this step (e.g., model forecasting and control calculations) is justified and supported by the increasing capabilities of modern computing systems used by the central control authority. Feedback from the fuel cell is then performed by updating the state of the model using the observer estimate that is provided by the sensors at discrete time instances. The model-based networked output feedback controller is then implemented as follows:

$$
\begin{aligned}
u(t) & =K \widehat{x}(t), t \in\left[t_{k}, t_{k+1}\right) \\
\dot{\bar{x}}(t) & =(\widehat{A}-L C) \bar{x}(t)+\widehat{B} u(t)+L y(t), t \in\left[t_{k}, t_{k+1}\right) \\
\dot{\hat{x}}(t) & =\widehat{A} \widehat{x}(t)+\widehat{B} u(t), t \in\left[t_{k}, t_{k+1}\right) \\
\widehat{x}\left(t_{k}\right) & =\bar{x}\left(t_{k}\right), k=0,1,2, \cdots
\end{aligned}
$$

where $\widehat{x}$ is an estimate of $x$. The model state is used by the controller so long as no information is transmitted over the network, but is updated (or re-set) using the observer estimate whenever it becomes available from the network.

A. Characterizing the minimum allowable communication rate over the network

Our first objective is to determine the largest update period that guarantees plant stability. This corresponds to the minimum rate at which the observer estimates need to be collected from the SOFC plant and transmitted to the controller over the network. To this end, we define the augmented state vector $\xi(t)=\left[x^{T}(t) \bar{x}^{T}(t) e(t)\right]^{T}$, where the estimation error is given by $e(t)=\bar{x}(t)-\widehat{x}(t)$, and let $t_{k}, k=0,1,2, \cdots$, be the instants when the model states are updated such that the update period $t_{k+1}-t_{k}=h$ is constant (generalizations to the problem of time-varying update periods are possible and the subject of other research work). Then it can be shown that the closed-loop system of Eqs.5-7 is of the form:

$$
\dot{\xi}(t)=\Lambda_{o} \xi(t), t \in\left[t_{k}, t_{k+1}\right), \xi\left(t_{k}\right)=\left[\begin{array}{c}
x\left(t_{k}\right) \\
\bar{x}\left(t_{k}\right) \\
0
\end{array}\right],
$$

where $e\left(t_{k}\right)=0$, for $k=0,1,2, \cdots$, since the model states are updated at $t_{k}$, and

$$
\Lambda_{o}=\left[\begin{array}{ccc}
A & B K & -B K \\
L C & \widehat{A}-L C+\widehat{B} K & -\widehat{B} K \\
L C & -L C & \widehat{A}
\end{array}\right]
$$

It can also be shown (see [20], [21]) that the system described by Eq.8 with initial condition $\xi\left(t_{0}\right)=$ 
$\left[x^{T}\left(t_{0}\right) \bar{x}^{T}\left(t_{0}\right) 0\right]^{T}=\xi_{0}$ has the following response:

$$
\xi(t)=e^{\Lambda_{o}\left(t-t_{k}\right)}\left(I_{o} e^{\Lambda_{o} h}\right)^{k} \xi_{0}, \text { for } t \in\left[t_{k}, t_{k+1}\right)
$$

with $t_{k+1}-t_{k}=h$, where $I_{o}=\left[\begin{array}{ccc}I & O & O \\ O & I & O \\ O & O & O\end{array}\right]$, and that a necessary and sufficient condition for the stability of this system is to have all the eigenvalues of the test matrix $M(h)=I_{o} e^{\Lambda_{o} h}$ restricted inside the unit circle. By examining the above expressions, it can be seen that the eigenvalues of $M$ depend on the mismatch between the model and the plant, the controller and observer gains, and the update period. To investigate the effect of model uncertainty on the stability of the networked SOFC plant, we consider as an example parametric uncertainty in $C p_{H_{2}}$ and define $\delta_{1}=\left(C p_{H_{2}}^{m}-C p_{H_{2}}\right) / C p_{H_{2}}$, where $C p_{H_{2}}^{m}$ is a nominal value used in the model, as a measure of model accuracy (any other set of uncertain parameters can also be considered and analyzed in a similar fashion). Figures
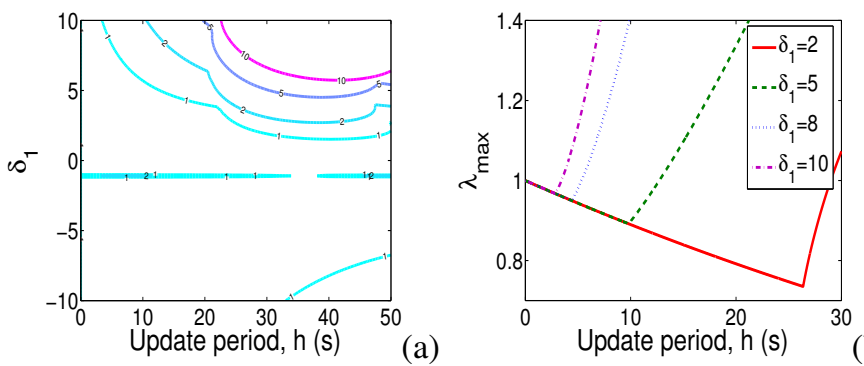

Fig. 2. Plot (a): Dependence of $\lambda_{\max }$ on plant-model mismatch for various update periods under output feedback control. Plot (b): Maximum stabilizing update period using different compensating models.

2(a)-(b) depict the dependence of the maximum eigenvalue magnitude, $\lambda_{\max }$, on both $\delta_{1}$ and the update period. In the contour plot (a), the area enclosed by the unit contour lines represents the stability region of the linearized plant. As expected, the range of tolerable parametric uncertainty shrinks as the update period is increased. The predictions of Figure 2(a) are further confirmed by the closed-loop temperature and power profiles in Figure 3 which show that the plant is stable when operated inside the unit contour zone $\left(\delta_{1}=5, h=8 \mathrm{~s}\right)$, and unstable when operated outside this region $\left(\delta_{1}=5, h=12.8 \mathrm{~s}\right)$.
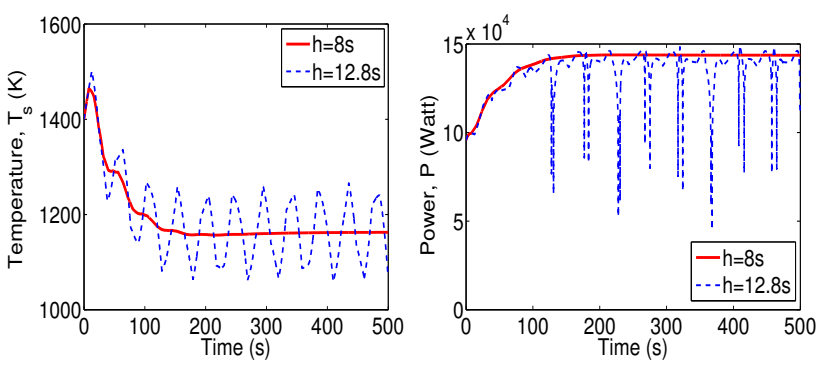

Fig. 3. Fuel cell stack temperature and power output under the networked output feedback control system with model uncertainty $\delta_{1}=5$ and different update periods.
Figure 2(b) shows the maximum eigenvalue magnitude versus the update period for different values of $\delta_{1}$. As expected, the maximum allowable update period decreases as the size of model uncertainty increases. It is clear that a model-based control scheme with a relatively accurate model can yield a larger update period.

\section{$B$. Incorporating performance considerations}

In addition to stability considerations, the performance of the networked fuel cell plant under disturbances is of major concern. Our objective in this section is to assess the performance of the networked closed-loop system under disturbances and characterize its dependence on the update period to determine a suitable communication rate that ensures minimal influence of the disturbances. To this end, we can re-write the linearized plant in the following form:

$$
\begin{aligned}
\dot{x} & =A x+B u+E w \\
y & =C x \\
z & =F x+G u
\end{aligned}
$$

where $w$ is the disturbance input and $z$ is the performance output signal of interest. After some manipulations, the networked closed-loop system can be formulated as:

$$
\begin{aligned}
\dot{\xi}(t) & =\Lambda \xi(t)+H w(t), t \in\left[t_{k}, t_{k+1}\right) \\
\xi\left(t_{k}\right) & =\left[\begin{array}{c}
x\left(t_{k}\right) \\
\bar{x}\left(t_{k}\right) \\
0
\end{array}\right], k=0,1,2, \cdots, h=t_{k+1}-t_{k} \\
z(t) & =N \xi(t)
\end{aligned}
$$

where $H=\left[\begin{array}{lll}E^{T} & O & O\end{array}\right]^{T}$ and $N=\left[\begin{array}{lll}F & G K & -G K\end{array}\right]$. Following [22], we use the extended $\mathrm{H}_{2}$ norm as the performance index. This is basically an $\mathrm{H}_{2}$-like norm that is suitable for analyzing periodic systems and captures the 2-norm of the performance output when an impulse disturbance is introduced in the input at $t=t_{0}$ (see [22] for the theoretical details and for other types of performance measures that can be used). In this case, the closed-loop response of the system to an impulse disturbance $w=$ $\delta\left(t-t_{0}\right)$ can be expressed explicitly as:

$$
z(t)=N e^{\Lambda_{o}\left(t-t_{k}\right)}\left(I_{o} e^{\Lambda_{o} h}\right)^{k} H, t \in\left[t_{k}, t_{k+1}\right)
$$

and the extended $\mathrm{H}_{2}$ norm, $\|G\|_{H_{2}}$, is given by:

$$
\|G\|_{H_{2}}=\operatorname{trace}\left(H^{T} X H\right)^{1 / 2}
$$

where $X$ is the solution of the discrete Lyapunov equation: $M(h)^{T} X M(h)-X+W_{o}(0, h)=0$ with $M(h)=I_{o} e^{\Lambda_{o} h}$, and $W_{o}(0, h)$ is the observability Gramian computed as $W_{o}(0, h)=\int_{0}^{h} e^{\Lambda_{o}^{T} t} N^{T} N e^{\Lambda_{o} t} d t$.

To test the performance of the networked control system, we initialize the closed-loop SOFC plant at the desired setpoint and introduce a unit impulse disturbance in the inlet flow rate of air, $q_{\mathrm{O}_{2}}^{i n}$. The fuel cell stack temperature $T_{s}$ is chosen as the performance output. In Figure 4(a), we plot the extended $\mathrm{H}_{2}$ norm of the system as a function of the update period when a model of the plant with uncertainty $\delta_{1}=5$ is embedded in the controller and an 
observer of the form of Eq.6 is embedded in the sensor. It can be seen that the optimal update period that minimizes the size of the extended $\mathrm{H}_{2}$ norm (and hence minimizes the effect of the disturbance on the stack temperature and ensures fastest recovery from the disturbance) occurs near $h=8 s$ (note that as expected this is less than the maximum allowable update period needed for stability) and that the performance degrades as the update period gets smaller. The performance also degrades as $h$ increases. Notice that in the limit as $h$ approaches zero, the value of the extended $\mathrm{H}_{2}$ norm approaches the value of the usual $\mathrm{H}_{2}$ norm for the non-networked (continuous) plant. The predictions of Figure 4(a) are confirmed by the stack temperature, power output and inlet fuel flow rate profiles shown in Figures 4(b)-(d) which show that the closed-loop system under $h=5 \mathrm{~s}$ exhibits a better response (faster recovery from the disturbance) than the one obtained at $h=12 \mathrm{~s}$. This is consistent with the fact that the value of the extended $\mathrm{H}_{2}$ at $h=5 \mathrm{~s}$ is smaller than it is at $h=12 \mathrm{~s}$.
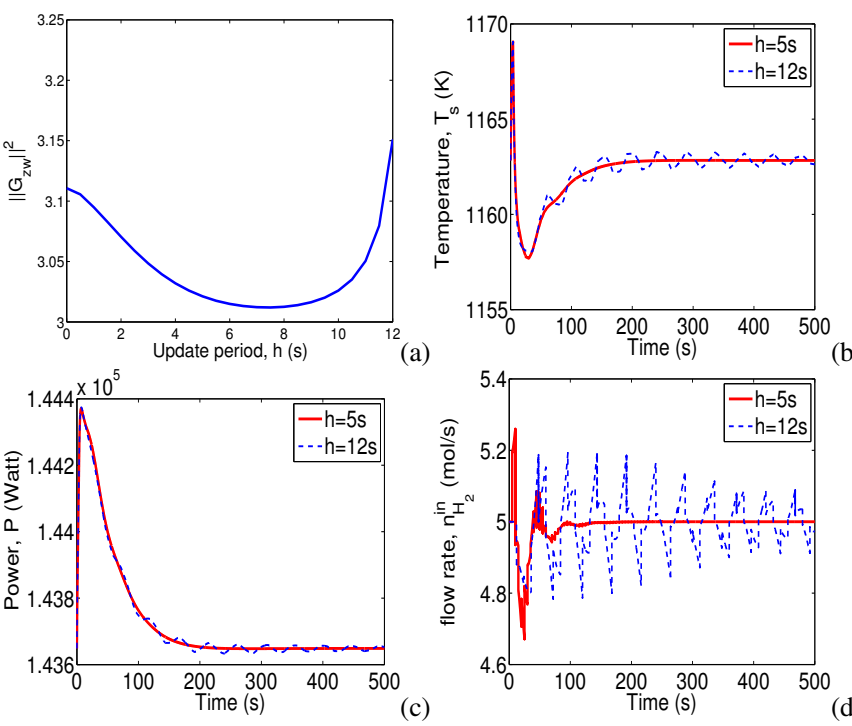

Fig. 4. Plot (a): Dependence of $\|G\|_{H_{2}}$ on the update period using a model with uncertainty $\delta_{1}=5$ under output feedback control. Plots (b)(d): Closed-loop stack temperature, power output and inlet fuel flow rate profiles under the networked control system for different update periods, when impulse disturbance is introduced in the inlet air flow rate.

\section{CONCluding REMARKS}

In this paper, we explored the application of concepts and techniques from networked control systems to address the problem of managing distributed energy resources over a bandwidth-constrained communication network. As a model system, we considered a solid oxide fuel cell plant and implemented a resource-aware networked control strategy that enforces the desired stability and performance properties with minimal communication between the fuel cell sensors and the controller over the network. The results were illustrated through numerical simulations. Extensions of the results to account for other network limitations, such as network-induced delays and real-time scheduling constraints, are under current investigation.

\section{REFERENCES}

[1] A. B. Lovins et al, Small Is Profitable: The Hidden Economic Benefits of Making Electrical Sources the Right Size. Rocky Mountain Institute, 2002.

[2] M. L. Wald, "Energy to count on: Fuel cells tapped as power source for computer systems," New York Times, August 17, 1999.

[3] K. Tomsovic and T. Hiyama, "Intelligent control methods for systems with dispersed generation," in Proceedings of IEEE Power Engineering Society Winter Meeting, Columbus, OH, 2001, pp. 913-917.

[4] F. Jurado and J. Saenz, "Adaptive control of a fuel cell-microturbine hybrid power plant," in Proceedings of IEEE Power Engineering Society Summer Meeting, Chicago, IL, 2002, pp. 76-81.

[5] M. Illindala and G. Venkataramanan, "Control of distributed generation systems to mitigate load and line imbalances," in Proceedings of IEEE Power Electronics Specialists Conference, Cairns, Australia, 2002, pp. 2013-2018.

[6] M. Marwali and A. Keyhani, "Control of distributed generation systems-part I: Voltages and currents control," IEEE Transactions on Power Electronics, vol. 19, pp. 1541-1550, 2004.

[7] R. Roberts, J. Brouwer, F. Jabbari, T. Junker, and H. Ghezel-Ayahg, "Control design of an atmospheric solid oxide fuel cell/gas turbine hybrid systems: Variable versus fixed speed has turbine operation," Journal of Power Sources, vol. 161, pp. 484-491, 2006.

[8] R. H. Lasseter, "Microgrids and distributed generation," Journal of Energy Engineering, vol. 133, pp. 144-149, 2007.

[9] K. Ro and S. Rahman, "Control of grid-connected fuel cell plants for enhancement of power system stability," Renewable Energy, vol. 28, pp. 397-407, 2003.

[10] A. Paradkar, A. Davari, A. Feliachi, and T. Biswas, "Integration of a fuel cell into the power system using an optimal controller based on disturbance accommodation control theory," Journal of Power Sources, vol. 128, pp. 218-230, 2004.

[11] K. Sedghisigarchi and A. Feliachi, "Dynamic and transient analysis of power distribution systems with fuel cells-part II: control and stability enhancement," IEEE Transactions on Energy Conversion, vol. 19, pp. 429-434, 2004.

[12] Z. Miao, M. A. Choudhry, R. L. Klein, and L. Fan, "Study of a fuel cell power plant in power distribution system - part II: Stability control," in Proceedings of IEEE Power Engineering Society General Meeting, St. Paul, MN, 2004, pp. 2226-2231.

[13] M. I. Marei, E. F. El-Saadany, and M. M. A. Salama, "A novel control algorithm for the DG interface to mitigate power quality problems," IEEE Transactions on Power Delivery, vol. 19, pp. 1384-1392, 2004.

[14] S. Barsali, M. Ceraolo, P. Pelacchi, and D. Poli, "Control techniques of dispersed generators to improve the continuity of electricity supply," in Proceedings of IEEE Power Engineering Society Winter Meeting, 2002, pp. 789-794.

[15] H. F. Wang, "Multi-agent co-ordination for the secondary voltage control in power-system contingencies," IEE Proceedings on Generation, Transmission and Distribution, vol. 148, pp. 61-66, 2001.

[16] L. M. Tolbert, H. Qi, and F. Z. Peng, "Scalable multi-agent system for real-time electric power management," in Proceedings of IEEE Power Engineering Society Summer Meeting, Vancouver, BC, Canada, 2001, pp. 1676-1679.

[17] K. J. P. Macken, K. Vanthournout, J. V. den Keybus, G. Deconinck, and R. J. M. Belmans, "Distributed control of renewable generation units with integrated active filter," IEEE Transactions On Power Electronics, vol. 19, pp. 1353-1360, 2004.

[18] A. L. Dimeas and N. D. Hatziargyriou, "Operation of a multiagent system for microgrid control," IEEE Transactions on Power Systems, vol. 20, pp. 1447-1455, 2005.

[19] A. M. Mursheda, B. Huang, and K. Nandakumara, "Control relevant modeling of planer solid oxide fuel cell system," Journal of Power Sources, vol. 163, pp. 830-845, 2007.

[20] L. A. Montestruque and P. J. Antsaklis, "On the model-based control of networked systems," Automatica, vol. 39, pp. 1837-1843, 2003.

[21] Y. Sun and N. H. El-Farra, "Quasi-decentralized model-based networked control of process systems," Comp. \& Chem. Eng., vol. 32, pp. 2016-2029, 2008.

[22] L. A. Montestruque and P. J. Antsaklis, "Performance evaluation for model-based networked control systems," in Netw. Emb. Sens. and Cntrl., LNCIS, vol. 331, P.J. Antsaklis, P. Tabuada (Eds.), SpringerVerlag, Berlin, 2006, pp. 231-249. 